\title{
Haptic feedback control of a smart wheelchair
}

\author{
Mohammed-Amine Hadj-Abdelkader ${ }^{\mathrm{a}, \mathrm{b}, *}$, Guy Bourhis ${ }^{\mathrm{a}}$ and Brahim Cherki ${ }^{\mathrm{b}, *}$ \\ ${ }^{a}$ Laboratoire d'Automatique Humaine et de Sciences Comportementales LASC, Université Paul Verlaine, \\ Metz, France \\ ${ }^{\mathrm{b}}$ Laboratoire d'Automatique de Tlemcen LAT, Université Abou Bekr Belkaïd, Tlemcen, Algeria
}

\begin{abstract}
The haptic feedback, which is natural in assistive devices intended for visually impaired persons, has been only recently explored for people with motor disability. The aim of this work is to study its potential, particularly for assistance in the driving of powered wheelchairs. After a review of the literature for the previous related work, we present the methodology and the implementation procedure of a haptic feedback control system on a prototype of a smart wheelchair. We will also describe the approaches utilized to determine the appropriate force feedback that will ensure a cooperative behaviour of the system, and we will detail the two haptic driving modes that were developed, namely the active and passive modes. Experiments on a real prototype were carried out to study the contribution of the method in powered wheelchair driving and to evaluate the interest of the force feedback on the control joystick of the wheelchair. They are discussed on the basis of performance measures.
\end{abstract}

Keywords: Smart wheelchair, motor disability, haptic feedback, human machine interaction, cooperative systems, laser sensor

\section{Introduction}

This work lies within the general scope of assistive technologies for disabled persons. Within this framework, the human-machine interaction is an essential feature and must be taken into account for every type of functionality (mobility assistance, manipulation aid, communication aid ...). In fact, as in every human-machine system, a dialogue must be established between the controlled system and the user. However, for persons with disabilities, this dialogue through conventional channels may become difficult, or even impos-

*Corresponding author: Mohammed-Amine Hadj-Abdelkader, Laboratoire d'Automatique Humaine et de Sciences Comportementales LASC, Université Paul Verlaine, 7 rue Marconi, Metz Technopole 57070, France. E-mails: ma_hadj@yahoo.fr, bourhis@univ-metz.fr and Brahim Cherki, Laboratoire d'Automatique de Tlemcen LAT, Université Abou Bekr Belkaïd, BP 230, Tlemcen 13000, Algeria; E-mail: B_cherki@yahoo.fr. sible, because of their motor and/or sensory deficiencies.

Three senses are naturally requested for the information feedback towards the user: vision, hearing and touch. This last one is broadly defined as "haptic" by including tactile and proprioceptive information (perception of our body, limbs position...) and kinaesthetic (feeling of the limbs movement). A sensory deficiency can be counterbalanced by the assistive device by calling upon another sense. For example, a mobility aid for blind people will use the haptic sense (blind cane) or the auditory sense (a telemetric cane that returns a sonic image of the near environment).

The transmission channel from the user to the assistive device may also be defective because of the motor deficiency of the person. We can then, in some cases, use an alternative to the classical muscle control by calling upon a voice recognition device. This assumes that the voice of the person is sufficiently 
reproducible and understandable. However, this control mode allows only for discrete commands. It is also possible to enhance and strengthen the motor control by haptic information at the human-machine interface: force feedback or tactile information. We present here a study of the potentialities of haptic feedback for assistive technologies intended for people with motor disabilities. We are especially interested in powered wheelchair control. Our matter is based on real experiments of powered wheelchair driving using the Microsoft force feedback joystick SideWinder $2^{\mathrm{TM}}$.

The composition of this paper is as follows. In Section 2, we present a review of the related work in the literature. In Section 3, the methodology of the proposed approach is described. The experimentation procedure and the results are then presented and discussed in Section 4.

\section{Related work}

Many research works about the teleoperation assisted by haptic feedback are described in the literature. These applications concern only users without disability but their conclusions could be extended to confirm the potential of the method. Thus, according to Sheridan, the force feedback in teleoperation is essential for some tasks but not for others [19]. For example, for a position control in free space, and particularly when the movements are slow, the haptic feedback has little advantages. It is however essential in handling or assembly tasks. In [2] simulation experiments were carried out for the teleoperation of a mobile base in a hostile environment. The authors found a significant decrease in the number of collisions by using a force feedback joystick compared to a conventional joystick. Nevertheless, the duration and length of paths were only slightly different from one situation to another. A similar experiment in [13] carried out using the 3D haptic device PHANToM ${ }^{\mathrm{TM}}$ of SensAble, limited to 2D, leads to the same conclusion: the haptic feedback decreases the number of collisions without increasing significantly the navigation time. However, performances measures are not enough to confirm the benefits of haptic feedback: in [12], measures of mental workload during the teleoperation of a helicopter show that some force feedback functions improve performances but significantly increase the mental workload.

\subsection{Haptic in rehabilitation engineering}

The first applications of haptic in rehabilitation engineering have been related naturally to the compensation of visual impairments, particularly with the invention of the Braille alphabet in the 19th century. Software interfaces associated with force and/or tactile feedback have appeared much more recently allowing a haptic rendering and sensations of forms designed on a computer screen. Applications of this principle are very varied: exploration of geographical charts, electrical circuits, mathematical curves, graphic documents on the web $[1,21] \ldots$ The force feedback has also been used for mobility assistance for persons with multiple disabilities, combining visual and motor impairments: the SPAM prototype (Smart Power Assistant Module) increases the resistance of the pushrims of a manual wheelchair according to the obstacles proximity [20]. In the same way, the powered wheelchair described in [14] returns information about the environment but, this time, through a force feedback joystick.

In the field of assistive technologies for persons with motor disabilities, the haptic feedback has become a subject of study only recently. Let's notice that the force feedback is less natural in this application context. Two issues seem to capture the researchers' attention. The first relates to the access to computer for people with motor disability, who experience troubles when using conventional pointing devices such as the mouse. Experiments on a panel of persons with disabilities have shown that a force feedback interface could significantly improve the performances in a pointing task [10]. These results are corroborated by a study described in [17] bearing on a group of 10 persons with motor disability. Moreover, the modelling of the coupling between the user and the haptic interface has shown that it is thus possible to remove the pathological tremor of some disabled persons [15]. The second issue, to which our work adheres more specifically, relates to the driving assistance of powered wheelchairs.

\subsection{Haptic and powered wheelchairs}

For many potential users, powered wheelchair control remains indeed difficult, or even impossible, because of their severe motor disability [7]. Some research teams have endeavoured to remedy this situation by developing "smart" wheelchairs [4]. The "intelligence" of the wheelchair can be defined as 
the ability to perceive the external environment and to retrieve relevant information in order to carry out autonomous or semi-autonomous movements: obstacle avoidance, doors passing, docking, automatic courses ... However, the wheelchair control in automatic mode brings up two major problems: a technical problem and a psychological problem. From a technical viewpoint, a perfect reliability of an automatic motion involves the use of a variety of sophisticated environmental sensors and a heavy data-processing that are not compatible with the cost requirements of such an application. From a psychological viewpoint, many potential users, on one hand, are apprehensive to let the whole movement control to the machine, and on the other hand, want to use their residual driving abilities as much as possible. A method to overcome these two problems is to introduce a force feedback on the control joystick. This force feedback is function of the obstacles proximity. We can then talk about an "assisted" control mode: the wheelchair control is entirely under the sole responsibility of the user, the machine, as a movement supervisor, provides him simply with haptic information to enhance the natural visual feedback. Within this context, the technical and psychological limitations of the automatic mode do not appear anymore. However, it remains to be demonstrated that the driving performances will be improved to a significant degree compared to the conventional driving mode of powered wheelchairs.

Few works of this type are referenced in the literature. In [6] a joystick was specifically designed to test in a fully modelled environment a "passive" force feedback algorithm (the joystick resists to movements towards obstacles) and an "active" force feedback algorithm (the joystick moves the wheelchair away from obstacles). The "active" algorithm has proved to be more effective. It has been tested using a virtual reality system on 5 persons with disability [16]: for 4 of them, the number of collisions in a test course has decreased, compared to driving without force feedback. In [9], the authors describe an "active" type algorithm based on the modified potential field method: to overcome the difficulty of passing doors with this method, the authors consider only the obstacles lying within $\pm 30^{\circ}$ from the wheelchair moving direction to calculate the repulsive force. Note that the authors have only tested a simple door passing task with this algorithm on a computer simulated mobile base. However, the application of this method on a real wheelchair will be quite different because, first, of the real experimenting conditions that include many constraints such as wheel sliding and the wheelchair's big dimensions. And, second, the fact that there is a person sitting on the wheelchair will add an extra inertia to the system which will completely change its dynamic characteristics. These works were all performed by computer simulation, except for [6] who tested the wheelchair's driving performances using only dead reckoning measures in a previously mapped and fully modelled environment. Thus, our goal is to investigate the contribution of haptic feedback to the driving performances in real driving conditions, with the use of environmental sensors and without pre-programmed paths.

\section{Methodology}

\subsection{Introduction}

Our aim is to design a new control mode for powered wheelchairs based on haptic feedback: depending on obstacles proximity, we apply a force on the device to help the pilot moving towards the closest free space to the direction he has chosen. This requires the use of telemetry sensors on the mobile base: for this purpose, we used a prototype of smart wheelchair developed in the VAHM project [4] that is outfitted with a Hokuyo ${ }^{\mathrm{TM}}$ laser rangefinder allowing obstacles detection within a $240^{\circ}$ sector and up to 4 meters away. This sensor is located at the front of the wheelchair at about $30 \mathrm{~cm}$ from the floor. It can sense all the obstacles on his horizontal plane and thus give an idea about the shape of the environment. However, if some obstacles are missed, it is not critical for the task in hand since we are working on driving assistance instead of the entirely automatic motion, the driving security being indeed under the control of the driver in all cases.

Given the difficulty of performing driving tests on a real wheelchair for people with disabilities, it is preferred to achieve these experiments on a driving simulator. Thus, we initially conducted a series of simulation experiments with a panel of persons without disability. The results are described in $[5,18]$. We particularly noticed that in certain environmental areas, such as door passages, the use of a force feedback improves the driving performances.

In the second step, we implemented this method on the VAHM wheelchair to evaluate it in real driving conditions. This implementation and the experiment results are presented in the following sections. These 


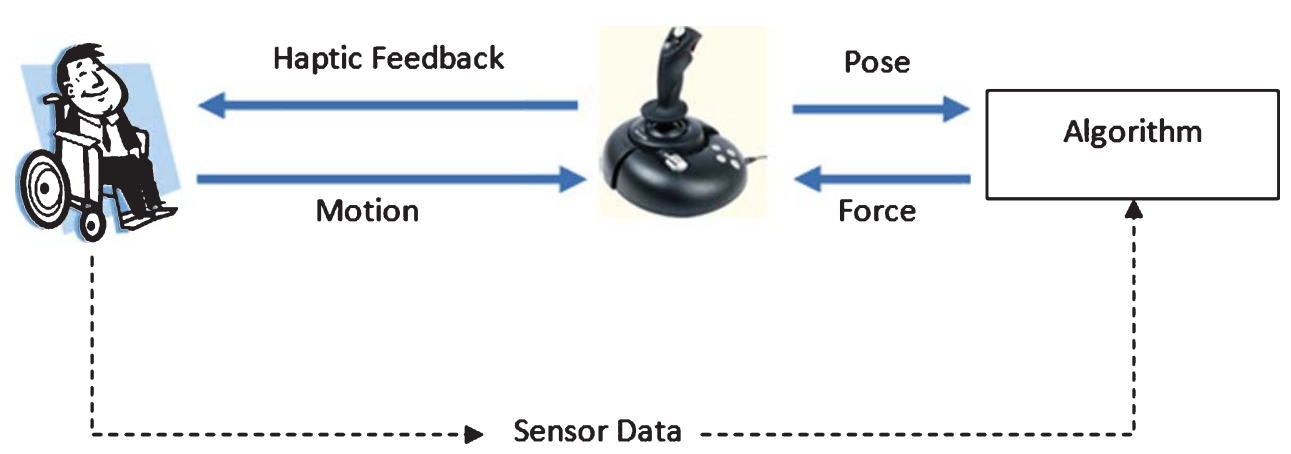

Fig. 1. The haptic control scheme of the system.

experiments on the real prototype allowed us to perceive the limitations of the previously developed simulator and will help us improving it. This way, we will get a reliable tool to analyze this technique with persons with disabilities.

Figure 1 shows the haptic control scheme of the system. The main steps of this control process can be summarised in the following points:

- First, the user applies a motion on the joystick in order to command the wheelchair.

- The magnitude and the orientation angle of this motion are then retrieved from the joystick and sent to the force computation algorithm which will use them as a set point for his calculations.

- Along with the sensor measurements, the algorithm determines the free directions in the environment, and applies a force on the joystick depending on the obstacle settings and on the selected driving mode.

- A haptic feedback is perceived by the user, who will adapt his motion on the joystick either by following the algorithm's chosen directions if they match his intentions or by adapting his force to keep the same heading even though it is not a suitable algorithm's choice. This may happen, for example, in the case the user wants to dock at a table; the algorithm in this case will tell him that this may not be a free direction, without preventing him from achieving his goal.

Notice that, in a haptic control process, the computations should be performed at a relatively high frequency in order to ensure a continuous interaction with the human. Thus, the algorithms that will be used must not require heavy calculus nor handle a huge amount of data.
The haptic information in this context could be very useful for some persons with motor disability who have difficulties moving their heads and cannot see all the obstacles in the environment. The advantage of this control method is that the control device is bilateral: it allows the user to send commands and to perceive information at the same time. So the user does not need to switch his attention between several components to drive the wheelchair.

\subsection{Force feedback algorithm}

We used a grid-based method to compute the feedback forces. We followed the same steps as in the VFH method introduced by [3]. The VFH method was initially used for obstacle avoidance for autonomous mobile robots outfitted with ultrasonic sensors and dead reckoning. It allows taking account of the sensor's measuring errors by filling a Cartesian grid with certainty values. These values match the actual positions of obstacles. In this approach, we adapted the VFH method to the utilisation with the laser sensor, by computing an obstacle density within each cell of the Cartesian grid instead of certainty values, and we did not use the dead reckoning process to calculate the wheelchair displacement because of the biased estimation it induces. We also wanted our system to be adaptable on every type of powered wheelchairs and thus, we tried to make it less dependent of additional sensors. Furthermore, dead reckoning systems are not easy to implement on wheelchairs. Consequently, the Cartesian grid in our method will be reset and filled up over again at each control loop cycle.

In addition, the Cartesian grid was centred at the laser sensor's position instead of the wheelchair centre point to reduce the grid's uncovered area. However, at the end of the algorithm, the resulting directions will 
be converted to the wheelchair's control frame centred at the midpoint of the rear wheels axis. For this implementation, we chose a grid of $50 \times 50 \mathrm{dm}^{2}$ divided into cells of $1 \mathrm{dm}^{2}$ each as shown in Fig. 2 .

In each control loop cycle, we compute the force feedback as follows:

1) Reading measurement values from the laser rangefinder,
4) The grid is then split into $5^{\circ}$ sectors, as shown in Fig. 4. For each sector we compute the polar obstacle density that is the sum of the values of all cells within the same sector. We get thus a polar histogram of polar densities. To avoid large densities variations in the histogram, we apply the smoothing function (2), where h(i) is the polar obstacle density for the ith sector.

$$
h(i)=\frac{3 h(i-2)+4 h(i-1)+5 h(i)+4 h(i+1)+3 h(i+2)}{19}
$$

2) Filling up the Cartesian grid by incrementing the values of the cells that match an obstacle position. If there are many obstacle detections within the same cell, then its value will be incremented to match the number of detections. This allows us to have an indication of the obstacles density within each cell (Fig. 3),

3) Then we apply the potential field principle [11] to the grid, i.e. we suppose that each cell will apply a repulsive force on the wheelchair; the force magnitude is given by the formula $(1)$, where $\mathrm{c}(i, j)$ is the density value associated with the cell $(i, j)$, $\mathrm{d}(i, j)$ is the distance between the sensor and the cell, $\mathrm{a}$ and $\mathrm{b}$ are the coefficients we use to get $\mathrm{a}$ zero magnitude for the farthest cell.

$$
m(i, j)=c(i, j) .(a-b \cdot d(i, j))
$$

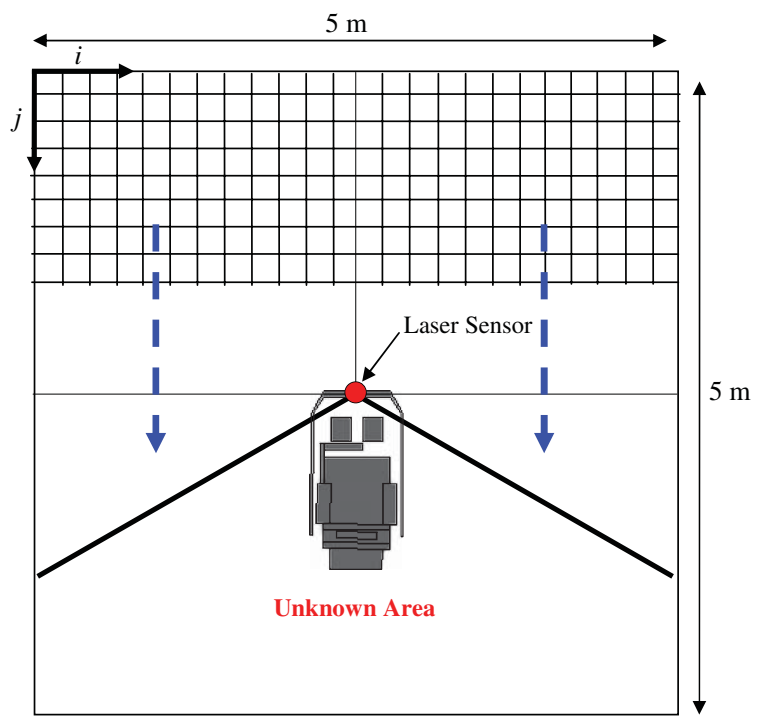

Fig. 2. The Wheelchair and the laser sensor in the Cartesian grid.
5) In the polar histogram, there are peaks showing the obstacles directions and valleys representing the free directions in the environment (Fig. 6). This distinction is made using an empirically chosen threshold. Notice that the selected valleys should be large enough in order to match the spaces that the wheelchair can easily get through. Hence, the valleys must be at least $25^{\circ}$ large (i.e. hold at least 5 sectors), which corresponds to a $0.9 \mathrm{~m}$ door passage at the farthest limit of the measured area on the Cartesian grid.

After the valleys detection, two different ways were used to send the force feedback to the joystick:

- Active force feedback: the force direction is the middle of the closest valley to the user's chosen direction. However, for each user the force magnitude should be adjusted. For safety reasons, the wheelchair motion must be started by the trigger of the joystick. This is due to the use of a positionsensing joystick that cannot sense forces. The user can stop the wheelchair simply by releasing the trigger.

- Passive force feedback: in this case, the joystick is hold at its central position with a medium strength resistive force. When the user drives towards a free direction, the force magnitude is reduced progressively to a fixed level, depending on whether the user is at the centre or at the border of the valley. However, when the user drives towards an obstacle (indicated by a peak on the histogram) then the resistive force magnitude is increased to push the user away to the free space. The wheelchair can be stopped by releasing the joystick (no trigger is needed). 


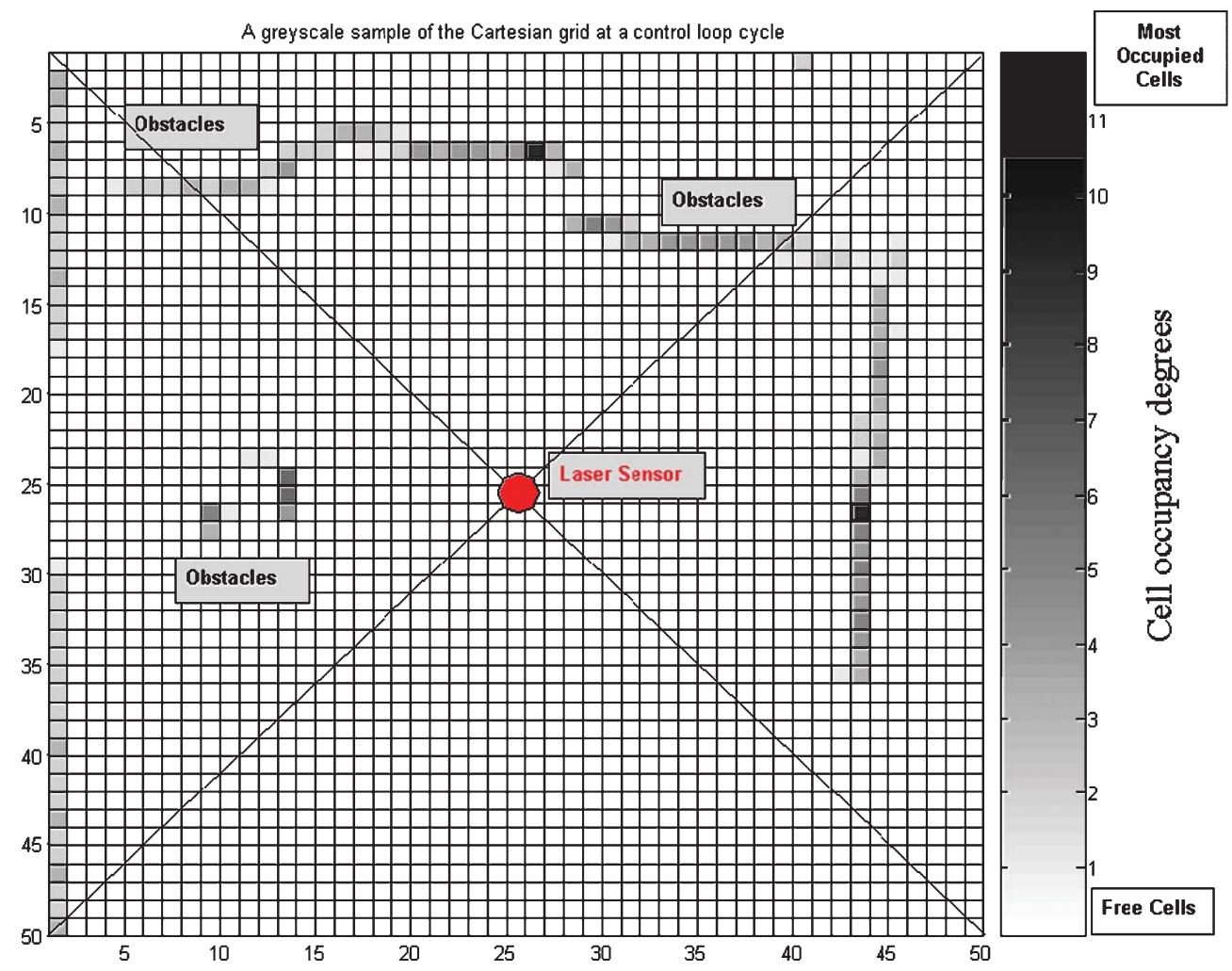

Fig. 3. Cartesian grid filled up with certainty values, displayed using greyscale.

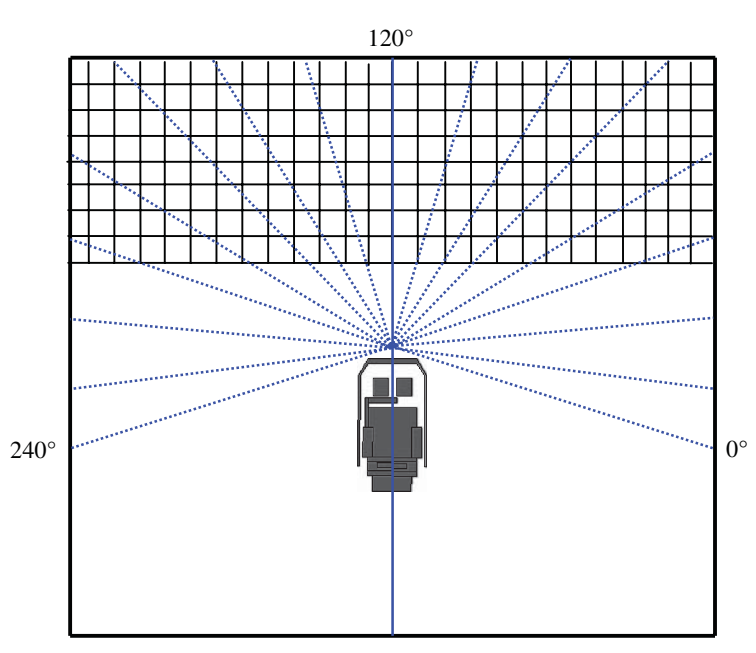

Fig. 4. Cartesian grid split into sectors.

\subsection{Force sending method}

After determining the resulting direction in the environment by the previously shown algorithms, a force is sent to the joystick in that direction. However, the joystick allows for many types of force feedback and we must find the most convenient way to send forces in order to ensure a cooperative behaviour, especially for the active mode. We found first that sending a constant force to the device does not allow the driver to have the entire control on the machine and that the joystick actions have become tough. A constant force on the joystick is indeed very hard to counter and is not a suitable solution for this kind of systems. After many trials, we agreed that the best way to apply forces is to produce a 'spring' effect on the joystick. Thus, the chosen direction will be the rest zone of a spring of a well adjusted stiffness. This way, the user will be able to choose easily another direction if he wants to, and will perceive enough strength on the joystick to figure out the positions of obstacles. Note that the spring effect stiffness was adjusted during the trail runs.

This type of effect was also applied to the passive mode because it allows the wheelchair control to be stable (i.e. it returns to a steady position when 


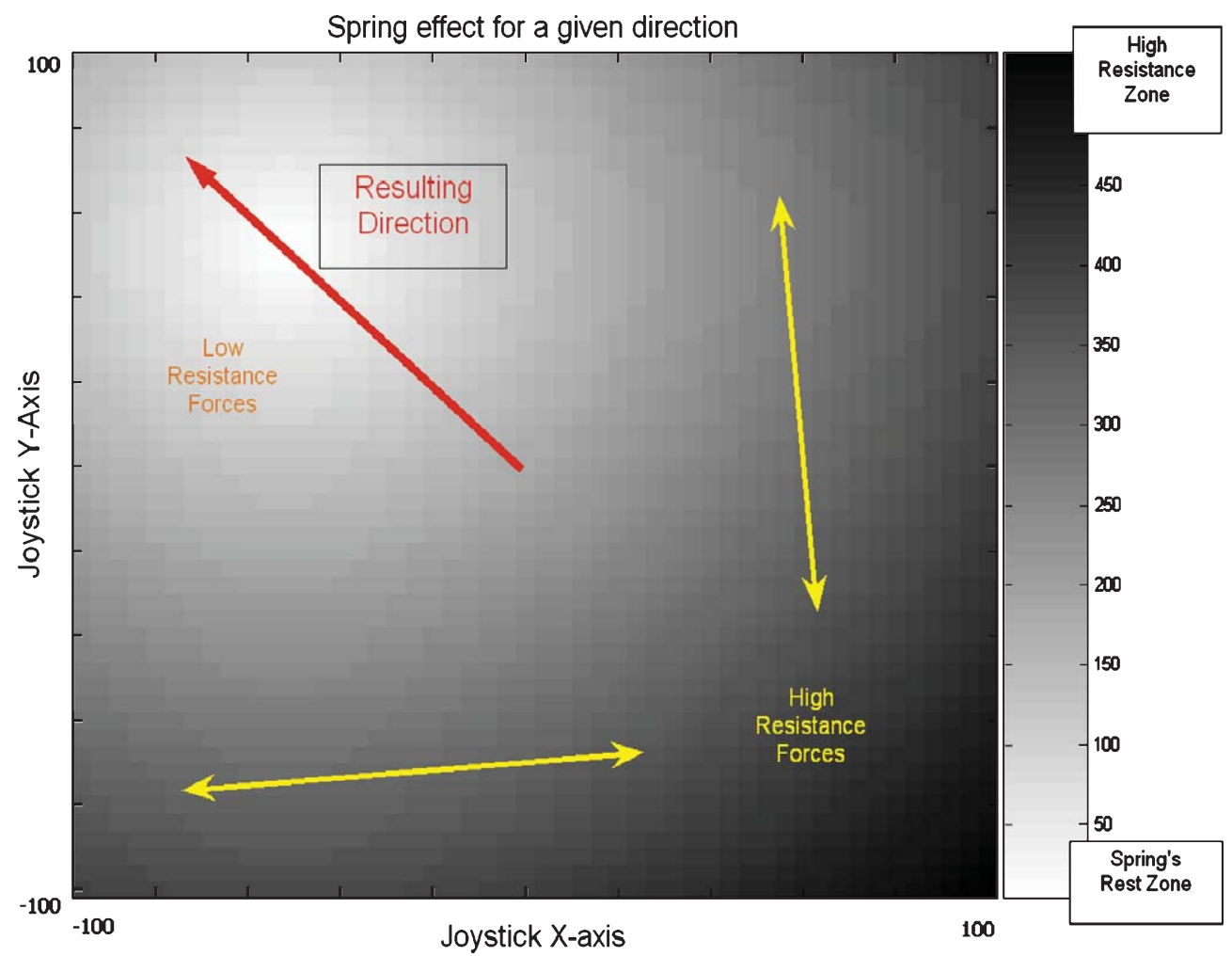

Fig. 5. Example of force application in the active mode.

there is no action on the system's actuator). However, an inertia effect was also manually implemented to make the spring's resistance appear gradually according to whether the applied direction is close to the algorithm's choice or not. Therefore, the system's behaviour will be entirely cooperative and will allow the user to have the full control on the wheelchair. Figure 5 show an example of force application in the active mode.

We also applied some control on the joystick's motion amplitude, such that the wheelchair' speed in the chosen direction will depend on the distance to obstacles in that direction. Thus, the speed of the vehicle will be at the maximum when the space in the direction of motion is entirely free, and it will decrease progressively if the sensor detects some obstacles in front of the wheelchair. Then, when the obstacles become too close, the algorithm will switch to another direction (supposed to be free) or will not send any haptic feedback if no free space is perceived.

\section{Experimentation and results}

To evaluate this approach, we asked a group of 10 valid and healthy users ( 9 men and 1 woman) to drive the wheelchair in an experimental environment, made up of 3 areas, involving different driving behaviours. Users were first given some trial runs, and then they started driving the wheelchair through the 3 areas. Each user began by driving without force feedback. After that, the force feedback was enabled and the user drove again using successively the passive and active algorithms. For all the users, the experiments were held on different sessions and distributed over several days in order to avoid biasing the results by learning effects.

To evaluate the driving performances, we recorded the course completion time (without counting the stopping time) and the number of collisions for each zone and each driving mode. The results presented here are the averages (for the completion times) and the sums 


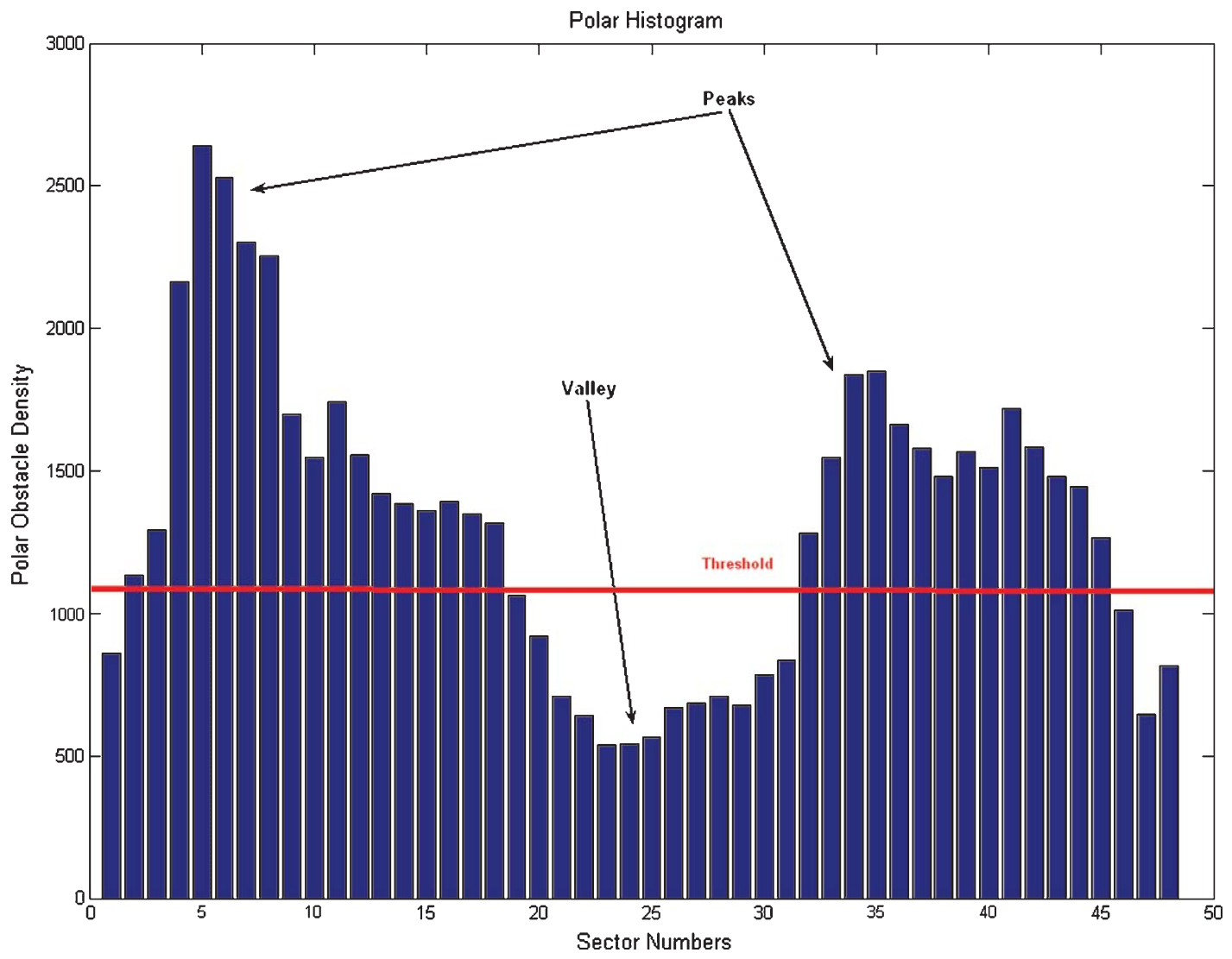

Fig. 6. The polar histogram and the valleys detection.

(for collisions) of measures taken on 4 courses for each user and each mode. For the active and passive force feedback algorithms, a record of the resulting direction and of the resistive force magnitude, respectively, allowed us to measure the algorithm's "follow-up rate". This rate is the percentage of driving time during which the user was applying a direction that belongs to the valley chosen by the algorithm. This will help us to assess the algorithm's accordance with the user choices and will allow us to estimate their efficiency. In addition, we used the TLX - Task Load Index - method [8] to evaluate the drivers' workload in each mode. This subjective questionnaire includes a user's estimation of the levels of concentration he reached, of the physical effort he spent, of the temporal constraints and the frustration degree he felt, and of the performance' level he accomplished. Each of these ratings is given by the user himself, on a scale from 0 to $100 \%$, according to his own appreciation. The higher will be the workload, the tougher was the driving task. Hence, we can determine the less laborious mode for the users.
For the active force feedback algorithm, more training was needed in order to adjust the force magnitude for each user. The tuning was made by varying the force magnitude, corresponding to the spring effect stiffness, till we get a value that allows for reasonable and relevant feedback. This adjusted force magnitude should help the user in the navigation task without forcing him to an unwanted direction or getting him frustrated. Notice that the obtained level of force was approximately the same for all the users.

The first zone of the course (Fig. 7) is made up of a $180^{\circ}$ corners combined with door passages of $0.9 \mathrm{~m}$ wide. Moreover, the corridors of this zone are relatively narrow $(1.35 \mathrm{~m}, 1.4 \mathrm{~m}, 1.5 \mathrm{~m})$ which makes the passing of corners very difficult. Notice that the wheelchair is about $1.2 \mathrm{~m} \times 0.8 \mathrm{~m}$ and that it needs a large manoeuvring area to steer correctly. The user starts driving from point D1 and travels to the point A1. The results for this area are shown in Table 1 . We notice first that the completion times for the passive and active modes were shorter than the completion times for the 

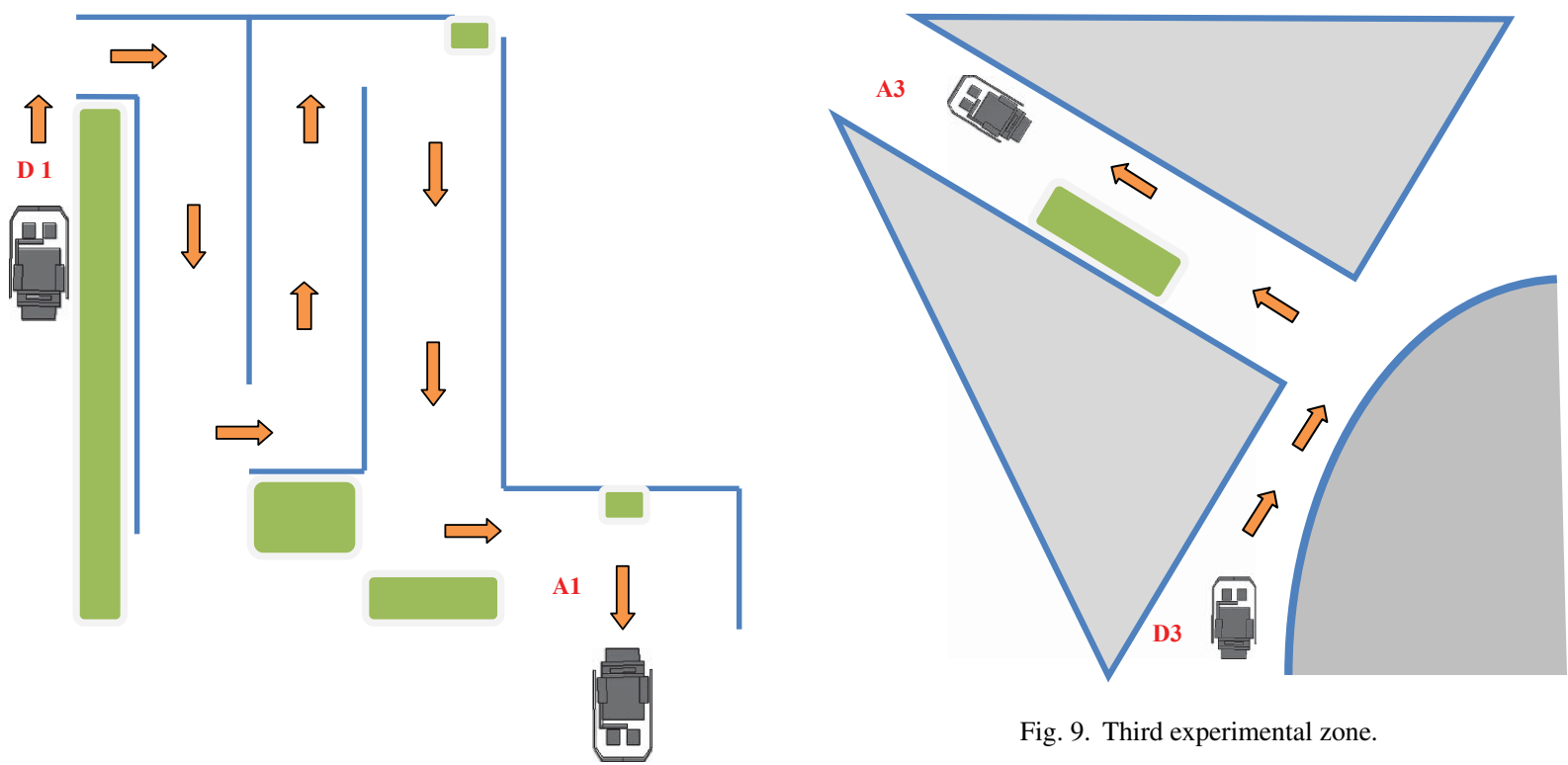

Fig. 9. Third experimental zone.

Fig. 7. First experimental zone.

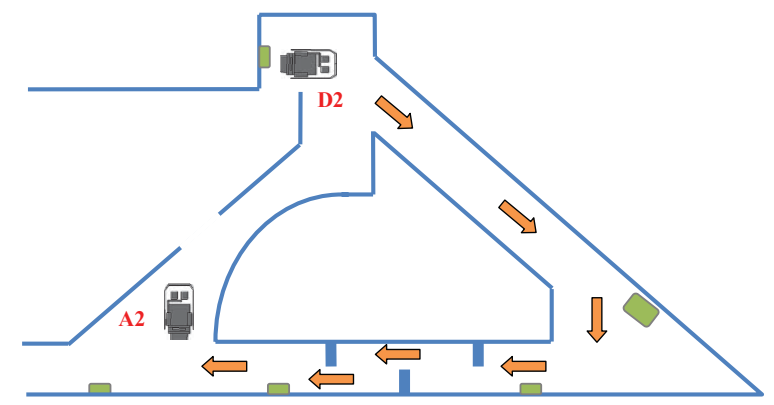

Fig. 8. Second experimental zone.

normal (without force) mode. An ANOVA analysis with a 0.05 alpha-level confirmed this observation. We noticed also that the number of collisions was reduced when the force feedback was applied, especially for the active algorithm. For the passive and active force feedback modes, the users admitted that the wheelchair driving was easier in the cluttered areas because they were able to recognize straightforwardly the free directions and to move through. The follow-up rates show that the compliance between the user and the algorithm was average in this zone.

The second zone of the course is mainly made up of 2 corridors of about $1.8 \mathrm{~m}$ wide, with 3 door passages of $0.9 \mathrm{~m}$ wide and some obstacles impeding manoeuvres nearby (Fig. 8). The starting point is D2,
Table 1

Time averages, collisions and follow-up rates for the first zone

\begin{tabular}{llccc}
\hline User & Driving mode & Times (s) & Collisions & $\begin{array}{c}\text { Follow-up } \\
\text { rates (\%) }\end{array}$ \\
\hline \multirow{4}{*}{1} & Without force & 40.38 & 2 & \\
& Passive & 35.83 & 1 & 60.34 \\
& Active & 32.76 & 0 & 66.27 \\
& Without force & 57.75 & 5 & \\
& Passive & 47.82 & 4 & 50.27 \\
& Active & 35.58 & 2 & 61.70 \\
& Without force & 40.54 & 4 & \\
3 & Passive & 39.32 & 3 & 51.85 \\
& Active & 41.82 & 0 & 58.88 \\
& Without force & 44.12 & 6 & \\
4 & Passive & 42.13 & 3 & 49.03 \\
& Active & 39.60 & 2 & 60.12 \\
& Without force & 41.36 & 0 & \\
5 & Passive & 35.82 & 1 & 57.59 \\
& Active & 33.22 & 0 & 68.53 \\
& Without force & 46.93 & 3 & \\
6 & Passive & 47.67 & 1 & 53.30 \\
& Active & 36.97 & 0 & 49.15 \\
& Without force & 40.69 & 0 & \\
7 & Passive & 39.04 & 0 & 49.15 \\
& Active & 38.84 & 0 & 50.36 \\
& Without force & 40.22 & 0 & \\
8 & Passive & 39.77 & 0 & 73.40 \\
& Active & 36.73 & 0 & 62.81 \\
& Without force & 40.18 & 3 & \\
9 & Passive & 40.51 & 0 & 64.35 \\
& Active & 36.89 & 0 & 62.99 \\
\multirow{4}{*}{10} & Without force & 44.23 & 4 & \\
& Passive & 41.91 & 2 & 46.93 \\
& Active & 38.45 & 1 & 51.90 \\
\hline \multirow{4}{*}{6} & & & &
\end{tabular}


Table 2

Time averages, collisions and follow-up rates for the second zone

\begin{tabular}{|c|c|c|c|c|}
\hline User & Driving mode & Times (s) & Collisions & $\begin{array}{c}\text { Follow-up } \\
\text { rates }(\%)\end{array}$ \\
\hline & Without force & 51.42 & 0 & \\
\hline \multirow[t]{3}{*}{1} & Passive & 47,02 & 0 & 84.00 \\
\hline & Active & 46.67 & 0 & 87.84 \\
\hline & Without force & 55.24 & 1 & \\
\hline \multirow[t]{3}{*}{2} & Passive & 52.99 & 1 & 75.04 \\
\hline & Active & 48.70 & 0 & 83.84 \\
\hline & Without force & 48.03 & 1 & \\
\hline \multirow[t]{3}{*}{3} & Passive & 46.63 & 0 & 81.24 \\
\hline & Active & 46.55 & 0 & 81.95 \\
\hline & Without force & 55.42 & 0 & \\
\hline \multirow[t]{3}{*}{4} & Passive & 54.19 & 1 & 72.16 \\
\hline & Active & 51.76 & 0 & 84.49 \\
\hline & Without force & 47.78 & 0 & \\
\hline \multirow[t]{3}{*}{5} & Passive & 44.95 & 0 & 85.71 \\
\hline & Active & 45.55 & 0 & 82.95 \\
\hline & Without force & 50.97 & 2 & \\
\hline \multirow[t]{3}{*}{6} & Passive & 51.54 & 1 & 76.43 \\
\hline & Active & 50.92 & 0 & 59.40 \\
\hline & Without force & 49.63 & 0 & \\
\hline \multirow[t]{3}{*}{7} & Passive & 49.31 & 0 & 72.64 \\
\hline & Active & 49.21 & 0 & 63.31 \\
\hline & Without force & 47.46 & 0 & \\
\hline \multirow[t]{3}{*}{8} & Passive & 48.13 & 0 & 88.70 \\
\hline & Active & 47.84 & 0 & 62.54 \\
\hline & Without force & 48.37 & 0 & \\
\hline \multirow[t]{3}{*}{9} & Passive & 48.67 & 0 & 81.10 \\
\hline & Active & 46.78 & 0 & 60.54 \\
\hline & Without force & 54.02 & 0 & \\
\hline \multirow[t]{2}{*}{10} & Passive & 52.40 & 0 & 71.40 \\
\hline & Active & 51.77 & 0 & 56.94 \\
\hline
\end{tabular}

the finishing point is $\mathrm{A} 2$, and the results are shown in Table 2. The shape of this zone was less complicated than the others, and thus the driving performances were nearly the same for all the users and all the modes. There were though some collisions in the normal and passive mode. We can particularly notice the high follow-up rates which indicate a good efficiency of the force feedback algorithms in finding free directions. According to the users, the level of frustration in this zone was less than in the others because there were not complicated situations.

The third zone of the course is smaller than the others, but it is mostly made up of 2 narrow passages (about $0.85 \mathrm{~m}$ wide) that are difficult to cross (Fig. 9). D3 is the starting point and A3 is the endpoint. There is also a door passage of $1.5 \mathrm{~m}$ wide that is hard to get through because it is located just after the first narrow passage. The results of this zone are shown in Table 3. As in the first zone, we found a decrease in the completion times that was statistically confirmed by a 0.05
Table 3

Time averages, collisions and follow-up rates for the third zone

\begin{tabular}{|c|c|c|c|c|}
\hline$\overline{\text { User }}$ & Driving mode & Times (s) & Collisions & $\begin{array}{c}\text { Follow-up } \\
\text { rates }(\%)\end{array}$ \\
\hline & Without force & 17.44 & 1 & \\
\hline \multirow[t]{3}{*}{1} & Passive & 16.04 & 1 & 69.84 \\
\hline & Active & 15.48 & 0 & 61.93 \\
\hline & Without force & 26.36 & 1 & \\
\hline \multirow[t]{3}{*}{2} & Passive & 20.54 & 0 & 54.36 \\
\hline & Active & 16.67 & 0 & 57.06 \\
\hline & Without force & 17.61 & 1 & \\
\hline \multirow[t]{3}{*}{3} & Passive & 17.31 & 0 & 53.12 \\
\hline & Active & 16.59 & 0 & 51.59 \\
\hline & Without force & 22.62 & 1 & \\
\hline \multirow[t]{3}{*}{4} & Passive & 21.03 & 1 & 48.79 \\
\hline & Active & 18.73 & 0 & 45.83 \\
\hline & Without force & 18.64 & 0 & \\
\hline \multirow[t]{3}{*}{5} & Passive & 17.30 & 1 & 65.04 \\
\hline & Active & 15.81 & 0 & 58.83 \\
\hline & Without force & 19.25 & 2 & \\
\hline \multirow[t]{3}{*}{6} & Passive & 20.64 & 1 & 39.60 \\
\hline & Active & 18.40 & 0 & 59.09 \\
\hline & Without force & 18.91 & 0 & \\
\hline \multirow[t]{3}{*}{7} & Passive & 18.72 & 0 & 40.02 \\
\hline & Active & 17.94 & 0 & 46.36 \\
\hline & Without force & 17.52 & 0 & \\
\hline \multirow[t]{3}{*}{8} & Passive & 17.91 & 0 & 68.63 \\
\hline & Active & 16.25 & 0 & 58.47 \\
\hline & Without force & 19.12 & 0 & \\
\hline \multirow[t]{3}{*}{9} & Passive & 20.72 & 1 & 53.72 \\
\hline & Active & 16.61 & 0 & 48.30 \\
\hline & Without force & 18.95 & 0 & \\
\hline \multirow[t]{2}{*}{10} & Passive & 19.53 & 1 & 45.87 \\
\hline & Active & 18.66 & 0 & 47.78 \\
\hline
\end{tabular}

alpha-level ANOVA analysis. The number of collisions was also reduced for the active mode and the follow-up rates were still close to the average.

Table 4 shows the results for the entire experimental environment. We can see that there were less collisions with the active force feedback algorithm than with the others, as shown in the former works shown by $[5,6]$. The follow-up rates were above the average most of the time. We should notice that several complicated shapes of the environment have needed many manoeuvres in order to avoid collisions. However, these constraints were not taken into account in the obstacle avoidance algorithm and generated situations where the resulting direction was not matching the user's choice. Notice also that a valley represents only $25^{\circ}$ among the possible $360^{\circ}$, which is less than $10 \%$ of the possible directions. This explains why the follow-up rates were not very high.

During these experiments, we used the TLX questionnaire to evaluate the drivers' workload at the end of 
Table 4

Time averages, collisions, follow-up rates and workloads for the whole itinerary

\begin{tabular}{|c|c|c|c|c|c|}
\hline$\overline{\text { User }}$ & Driving mode & Times (s) & Collisions & $\begin{array}{c}\text { Follow-up } \\
\text { rates }(\%)\end{array}$ & $\begin{array}{c}\text { Workload } \\
(\%)\end{array}$ \\
\hline & Without force & 109.24 & 3 & & 38.80 \\
\hline \multirow[t]{3}{*}{1} & Passive & 98.89 & 2 & 71.39 & 41.80 \\
\hline & Active & 94.91 & 0 & 72.02 & 24.73 \\
\hline & Without force & 139.35 & 7 & & 49.73 \\
\hline \multirow[t]{3}{*}{2} & Passive & 121.35 & 5 & 59.89 & 64.47 \\
\hline & Active & 100.95 & 2 & 67.53 & 42.07 \\
\hline & Without force & 106.18 & 6 & & 64.40 \\
\hline \multirow[t]{3}{*}{3} & Passive & 103.26 & 3 & 62.07 & 62.63 \\
\hline & Active & 104.96 & 0 & 64.14 & 66.87 \\
\hline & Without force & 122.16 & 7 & & 38.33 \\
\hline \multirow[t]{3}{*}{4} & Passive & 117.35 & 5 & 56.66 & 38.47 \\
\hline & Active & 110.09 & 2 & 63.48 & 40.60 \\
\hline & Without force & 107.78 & 0 & & 53.33 \\
\hline \multirow[t]{3}{*}{5} & Passive & 98.07 & 2 & 69.45 & 41.27 \\
\hline & Active & 94.58 & 0 & 70.10 & 41.73 \\
\hline & Without force & 117.15 & 7 & & 51.93 \\
\hline \multirow[t]{3}{*}{6} & Passive & 119.86 & 3 & 56.44 & 33.33 \\
\hline & Active & 106.29 & 0 & 55.88 & 30.67 \\
\hline & Without force & 109.23 & 0 & & 46.73 \\
\hline \multirow[t]{3}{*}{7} & Passive & 107.06 & 0 & 53.94 & 18.40 \\
\hline & Active & 105.99 & 0 & 53.34 & 14.93 \\
\hline & Without force & 105.19 & 0 & & 16.27 \\
\hline \multirow[t]{3}{*}{8} & Passive & 105.81 & 0 & 76.91 & 11.73 \\
\hline & Active & 100.82 & 0 & 61.27 & 17.47 \\
\hline & Without force & 107.66 & 3 & & 33.60 \\
\hline \multirow[t]{3}{*}{9} & Passive & 109.90 & 1 & 66.39 & 30.27 \\
\hline & Active & 100.28 & 0 & 57.28 & 32.33 \\
\hline & Without force & 117.20 & 4 & & 32.94 \\
\hline \multirow[t]{2}{*}{10} & Passive & 113.84 & 3 & 54.73 & 13.73 \\
\hline & Active & 108.87 & 1 & 52.21 & 24.07 \\
\hline
\end{tabular}

each series of tests. At the beginning the active mode seemed to be heavier than the others. But once the users were used to it, they felt more comfortable with this mode and gave it a good appreciation as we can see in Table 4 where, in most cases, the active mode has got smallest workload values. The force feedback has clearly brought a good contribution to the navigation task in different ways: the active mode allowed to reduce errors and to make the driving smoother, whereas the passive mode helped to decrease the arm tiredness for the drivers because of the adapted resistive force.

We tried also in these experiments to record the joystick commands in order to analyse the driver's actions on it and to compare the different driving modes in terms of comfort. We recorded thus the joystick magnitude and the angle differences at each control cycle and took the highest angular difference for each experiment. However, the results were almost the same in all the situations which leads us to suppose that there were no changes in the driving behaviour for these three modes.

Finally, we should note that the grid-based method for the determination of force feedback was initially used for small and fast mobile robots that do not need any manoeuvres during their motion. Moreover, this obstacle avoidance method does not include any path planning technique, thus the driver was supposed to handle the manoeuvres by himself. Nevertheless, this method allowed us to get an immediate response from the control system and was very useful once the good wheelchair orientation was found.

\section{Conclusion}

The experiments presented here were carried out to evaluate the contribution of force feedback in the wheelchair driving task. Although we could not carry them out with users with disabilities for safety reasons, they were still informative on the force feedback contribution to the driving enhancement in real conditions. Thus, they allowed us also to perceive the shortcomings and limits of the previously designed simulator described in $[5,18]$. Hence, we acquired a good knowledge to improve this simulator in order to perform experiments with disabled persons. With such a tool, a better evaluation of the technique could be achieved, as well as the evaluation of other works intended to assist and help people with motor disabilities. We think also that the choice between the passive and active feedback should be given to the user who can select the most convenient mode for himself. A good future perspective would be the use of other haptic devices than the classical joystick and to compare these different devices in terms of performances, acceptability and workload. Finally, all these approaches should respect the basic ergonomic and usability rules and should also be accepted by the conventional wheelchair users in order to find their place among the currently available rehabilitation techniques.

\section{Acknowledgements}

The authors would like to thank the LASC Laboratory members who contributed to this work by performing the experiments on the wheelchair. 


\section{References}

[1] N. Baptiste-Jessel, B. Tornil and B. Encelle, Using SVG and a force feedback mouse to enable blind people to access "graphical" Web based documents, ICCHP 2004, Paris, France, 2004, pp. 228-235.

[2] D.P. Barnes and M.S. Counsell, Haptic Communication for Remote Mobile Manipulator Robot Operation, Proceedings 8th Topical Meeting on Robotics and Remote Systems, Pittsburgh, PA, USA, 1999.

[3] J. Borenstein and Y. Koren, The Vector Field Histogram - Fast Obstacle Avoidance For Mobile Robots, IEEE Transactions on Robotics and Automation 7(3) (1991), 278-288.

[4] G. Bourhis, O. Horn, O. Habert and A. Pruski, Autonomous vehicle for people with motor disabilities, IEEE Robotics and Automation Magazine, Special issue on "Reinventing the wheelchair" 7(1) (2001), 20-28.

[5] G. Bourhis and M. Sahnoun, Assisted control for a smart wheelchair, 10th IEEE International Conference on Rehabilitation Robotics, ICORR'07, Noordwjick, The Netherlands, 2007, pp. 158-163.

[6] D.M. Brienza and J. Angelo, A force feedback joystick and control algorithm for wheelchair obstacle avoidance, Disability and Rehabilitation 18(3) (1996), 123-129.

[7] L. Fehr, W.E. Langbein and S.B. Skaar, Adequacy of power wheelchair control interfaces for persons with severe disabilities: A clinical survey, Journal of Rehabilitation Research and Development 37(3), 2000, pp. 353-360.

[8] S.G. Hart and L.E. Staveland, Development of NASA-TLX (Task Load Index): Results of experimental and theoretical research. Édité par P.A. Hancock N. Meshkati (Eds). Human mental workload, 1988, 139-183.

[9] J.P. Hong, O.S. Kwon et al., Shared-control and force reflection joystick algorithm for the door passing of mobile robot or powered wheelchair, IEEE TENCON (1999), 1577-1580.

[10] S. Keates, P. Langdon, J. Clarckson and P. Robinson, Investigating the use of force feedback for motion impaired users, 6th ERCIM Workshop "User Interfaces for all”, Florence, Italy, 2000, pp. 207-212.

[11] O. Khatib, Real-Time Obstacle Avoidance for Manipulators and Mobile Robots, IEEE International Conference on Robotics and Automation, St. Louis, Missouri, (1985), 500-505.

[12] T.M. Lam, H.W. Boschloo, M. Mulder and M.M. Van Paase, Effect of Haptic Feedback in a Trajectory Following Task with an Unmanned Aerial Vehicle, IEEE International Conference on SMC, The Hague, The Nederland, 2004, 25002506.

[13] S. Lee, G.S. Sukhatme, G.J. Kim and C. Park, Haptic Control of a Mobile Robot: A User Study, Presence: Teleoperators \& Virtual Environments 14(3) (2005), 345-365.

[14] R.C. Luo, C.Y. Hu, T.M. Chen and M.H. Lin, Force reflective feedback control for intelligent wheelchairs, IEEE Int Conf on Intelligent Robots and Systems (1999), 918-923.

[15] S. Pledgie, K. Barner, S. Agrawal and T. Rahman, Tremor suppression through force feedback, ICORR'99, Stanford, CA USA, 1999, 16-26.

[16] J.L. Protho, E.F. Lopresti and D.M. Brienza, An evaluation of an obstacle avoidance force feedback joystick, RESNA Annual Conference, 2000, pp. 447-449.

[17] D.W. Repperger, C.A. Phillips and T.L. Chelette, A study on spatially induced "virtual force" with an information theoretic investigation of human performance, IEEE Trans actions on Systems, Man and Cybernetics 25(10) (1995), 1392-1404.

[18] M. Sahnoun and G. Bourhis, Conception et simulation d'une commande à retour d'effort pour fauteuil roulant électrique, Sciences et Technologies pour le Handicap 1(2) (2007), 123-141.

[19] T.B. Sheridan, Human and machine haptics in historica perspective, Workshop on Human and Machine Haptics, Introduction to the book, Stanford University, CA, USA 1997.

[20] R. Simpson et al., A prototype power assist wheelchair that provides for obstacle detection and avoidance for those with visual impairments, Journal of NeuroEngineering and Rehabilitation 2(30), 2005.

[21] C. Sjöström and K. Rassmus-Gröhn, The sense of touch provides new computer interaction techniques for disabled people, Technology and disability 10(1) (1999), 45-52. 

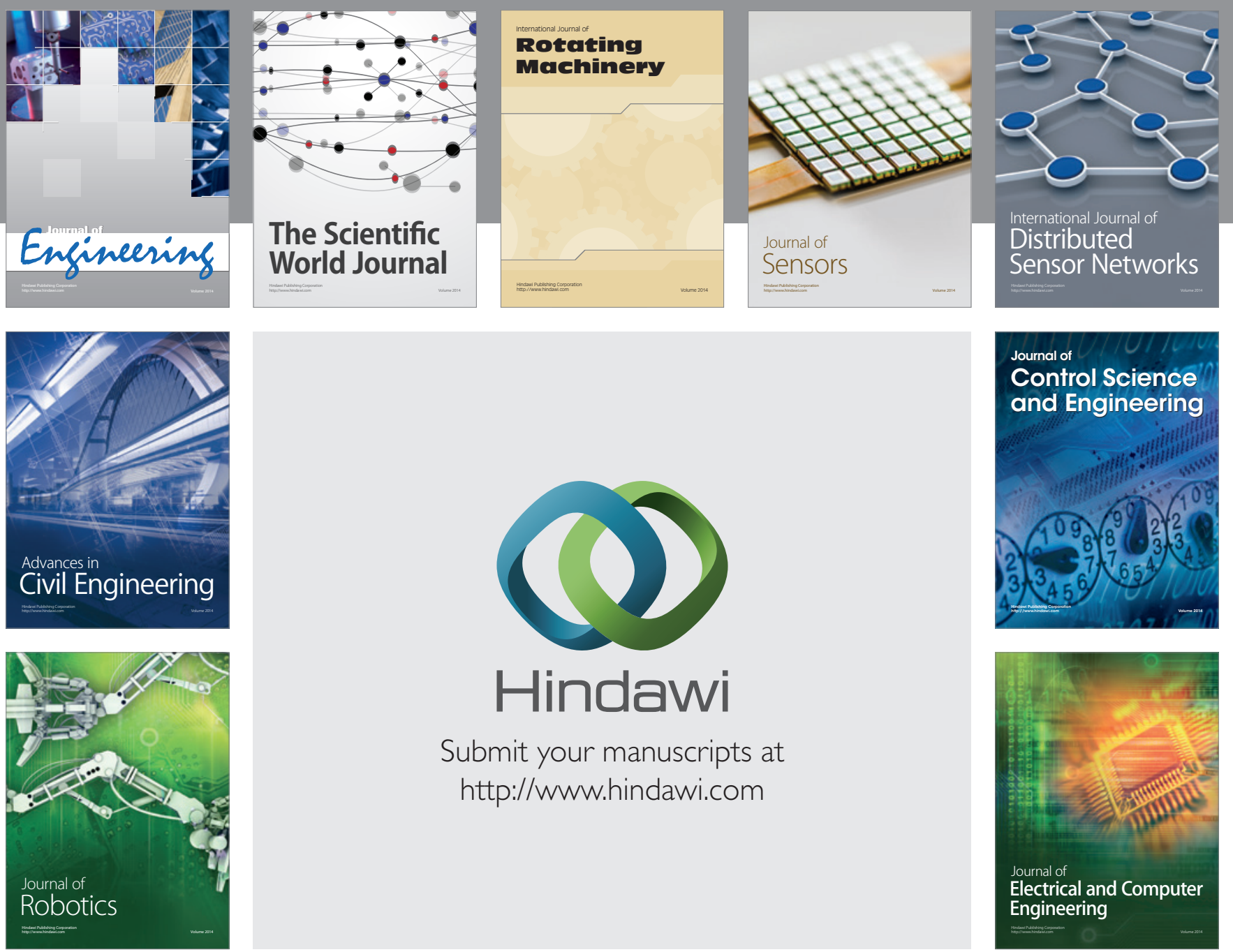

Submit your manuscripts at

http://www.hindawi.com
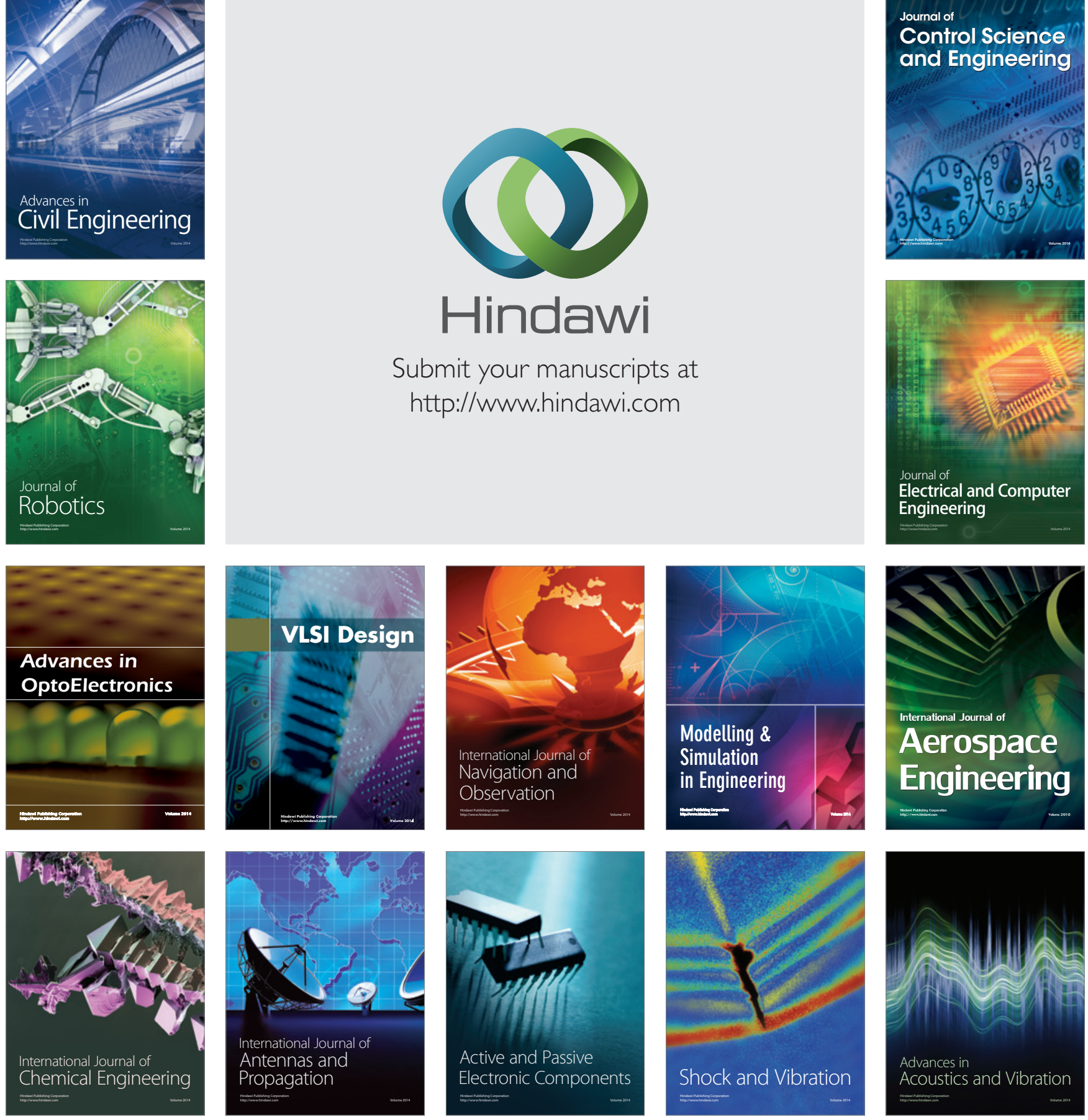